Article

\title{
Design of a Compliant Mechanism Based Four-Stage Amplification Piezoelectric-Driven Asymmetric Microgripper
}

\author{
Xiaodong Chen ${ }^{1}$, Zilong Deng ${ }^{1, *}$, Siya $\mathrm{Hu}^{1}{ }^{1}$, Jinhai Gao ${ }^{2}$ and Xingjun Gao ${ }^{1}$ \\ 1 College of Mechanical Engineering, Liaoning University of Petroleum and Chemical Technology, Fushun \\ 113001, China; c820083720@163.com (X.C.); Hsy2544138944@163.com (S.H.); gxj7976@163.com (X.G.) \\ 2 College of Mechanical Engineering and Automation, Northeast University, Shenyang 110000, China; \\ clj_242925@163.com \\ * Correspondence: dzl56801@sina.com; Tel.: +86-183-4031-1628
}

Received: 5 November 2019; Accepted: 20 December 2019; Published: 24 December 2019

\begin{abstract}
The existing symmetrical microgrippers have larger output displacements compared with the asymmetrical counterparts. However, the two jaws of a symmetrical microgripper are less unlikely to offer the same forces on the two sides of a grasped micro-object due to the manufacture and assembly errors. Therefore, this paper proposes a new asymmetric microgripper to obtain stable output force of the gripper. Compared with symmetrical microgrippers, asymmetrical microgrippers usually have smaller output displacements. In order to increase the output displacement, a compliant mechanism with four stage amplification is employed to design the asymmetric microgripper. Consequently, the proposed asymmetrical microgripper possesses the advantages of both the stable output force of the gripper and large displacement amplification. To begin with, the mechanical model of the microgripper is established in this paper. The relationship between the driving force and the output displacement of the microgripper is then derived, followed by the static characteristics' analysis of the microgripper. Furthermore, finite element analysis (FEA) of the microgripper is also performed, and the mechanical structure of the microgripper is optimized based on the FEA simulations. Lastly, experimental tests are carried out, with a 5.28\% difference from the FEA results and an $8.8 \%$ difference from the theoretical results. The results from theoretical calculation, FEA simulations, and experimental tests verify that the displacement amplification ratio and the maximum gripping displacement of the microgripper are up to 31.6 and $632 \mu \mathrm{m}$, respectively.
\end{abstract}

Keywords: microgripper; design; finite element analysis (FEA); piezoelectric drive; experimental verification

\section{Introduction}

With the rapid development of micro-/nano-technologies, precision processing, biological engineering, microelectronics, and aerospace [1-5], micro-manipulators with better operating performance are desired. As micro-operated end effectors, microgrippers are placed at the end of the arms of manipulators to interact with micro-objects, so they play a vital role in determining the success of micro-operation tasks. Compared with traditional rigid mechanisms, compliant mechanisms are more suitable for the design of microgrippers, due to their advantages such as no backlash, no requirement for lubrication, simplified manufacture, and low part count [6,7]. At present, a variety of microgrippers has been developed with different driving modes. The common driving modes of microgrippers are the piezoelectric drive [8,9], electrostatic drive [10,11], electrothermal drive [12,13], shape memory alloy (SMA) drive [14,15], pneumatic drive [16,17], etc. Compared with other driving modes, the piezoelectric drive has the advantages of small size, large output force, high sensitivity, and no gap. Therefore, it is more widely used as the actuators of microgrippers $[18,19]$. 
It is found from the current studies that the aim of the development of microgrippers is to increase the displacement amplification and improve the motion accuracy. The output displacement of a piezoelectric actuator is too small, usually tens of microns. In order to enlarge the strokes of piezoelectric actuators, displacement amplifiers are widely employed in piezoelectric driven systems. Existing micro-displacement amplifiers include the lever amplifier, bridge amplifier, and rhombus amplifier. Displacement amplifiers consist of a single lever, bridge, or rhombic and are categorized as single stage amplifiers. Amplifiers composed of multiple single stage amplifiers are called multi-stage amplifiers [20]. A multi-stage amplifier can largely increase the clamping stroke of a microgripper. The gripping accuracy of a microgripper mainly involves two issues: kinematic accuracy and output force of the gripper accuracy. The kinematic accuracy is mainly manifested by parallel gripping accuracy. When the gripping object is an irregularly, such as spherical or cylindrical, shaped component, the parallel gripping can realize stable gripping and assembly [21,22]. Parallel gripping can also reduce the stress concentration on the gripper when the gripping objects are fragile chip devices [23]. The accuracy of the output force of the gripper mainly depends on the control of the output force of the gripper of the grasping jaws. The existing gripping methods of microgrippers are symmetrical gripping and asymmetric gripping. Symmetrical gripping easily to damages the micro-object due to the uneven forces exerted on the micro-object from the left and right jaws of the gripper. One grasping jaw of an asymmetric microgripper is fixed to the ground, and the other grasping jaw is moveable. Therefore, the piezoelectric actuation system of a piezoelectric driven asymmetric microgripper is used to control the motion of one of the grasping jaws, which enables the motion control to be more accurate and stable. However, the output displacement of an asymmetric microgripper is small, which is usually half of the clamping stroke of the symmetrical counterpart.

Li et al. [24] designed a single stage symmetrical microgripper based on the principle of lever amplification to achieve a desired displacement amplification, but could not achieve parallel gripping. Cui et al. [25] designed a symmetrical microgripper based on the principle of lever amplification that could realize parallel gripping. However, the actual maximum output forces of the left and right jaws were different, $8.02 \mathrm{mN}$ and $9.24 \mathrm{mN}$, respectively. The output forces were different, so it was easy to destroy the microparts in the clamping process. Sun et al. [26] designed a two stage symmetrical microgripper based on the principle of lever amplification and triangle amplification, which realized parallel clamping of the jaws and high amplification. Wang et al. [27] designed a three stage symmetrical microgripper based on the principle of lever amplification and triangle amplification to further improve the amplification. However, the microgrippers designed by Sun et al. [26] and Wang et al. [27] could not achieve stable gripping. Koo et al. [28] designed a single stage asymmetric microgripper based on the principle of lever amplification, which realized the stable gripping of the jaws, but could not be clamped in parallel. Xing et al. [29] designed a single stage asymmetric microgripper based on the principle of lever amplification, which realized the parallel gripping of the jaws, but the amplification was small. Zhao et al. [30] designed a two stage asymmetric microgripper based on the principle of lever amplification, which realized the stable gripping of the jaws and improved displacement amplification. Based on the principle of lever amplification and triangle amplification, Liang et al. [31] designed a three stage asymmetric microgripper, which realized the parallel gripping, but the amplification was smaller than that of Li et al. [24], Sun [26], and Wang [27].

In summary, compared with symmetrical microgrippers, an asymmetric microgripper has more stable gripping performance. It is necessary to design a kind of piezoelectric driven microgripper with high displacement amplification and high stable gripping performance. In this paper, a new piezoelectric driven asymmetric microgripper is proposed with a high amplification ratio (31.6) and parallel gripping performance. 


\title{
2. Structure Design and Motion Principle of the Microgripper
}

\subsection{Structure Design of the Microgripper}

Figure 1 shows a diagram of the main view of the microgripper. The size of the mechanism was $68.8 \mathrm{~mm} \times 34.55 \mathrm{~mm} \times 5 \mathrm{~mm}$. The microgripper mainly consisted of a stacked piezoelectric (PZT) ceramic actuator (SPCA), an asymmetric right-circular flexure hinge, a rectangular flexure hinge, a grasping jaw, a double lever amplifier, a double bridge amplifier, a fixing hole, and a preload bolt. In order to further improve the clamping stroke and obtain larger output displacement, a four stage amplifier was designed, including a double bridge amplifier and a double lever amplifier. One end of SPCA was fixed with the displacement transmission mechanism (DTM) by the preload bolt, and the preload force on SPCA could be adjusted by the bolt. The double bridge amplifier and the lever amplifier were directly connected in series to obtain a larger displacement amplification. Rectangular hinges were employed in the double bridge amplifier due to the fact that rectangular hinges have large displacements, but small stress concentrations compared with other types of hinges [32-34].

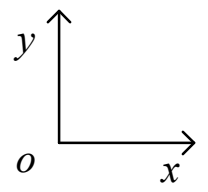

\section{Fixed jaw}

Moveable jaw

\begin{abstract}
Asymmetric
right-circular flexure hinge
\end{abstract}
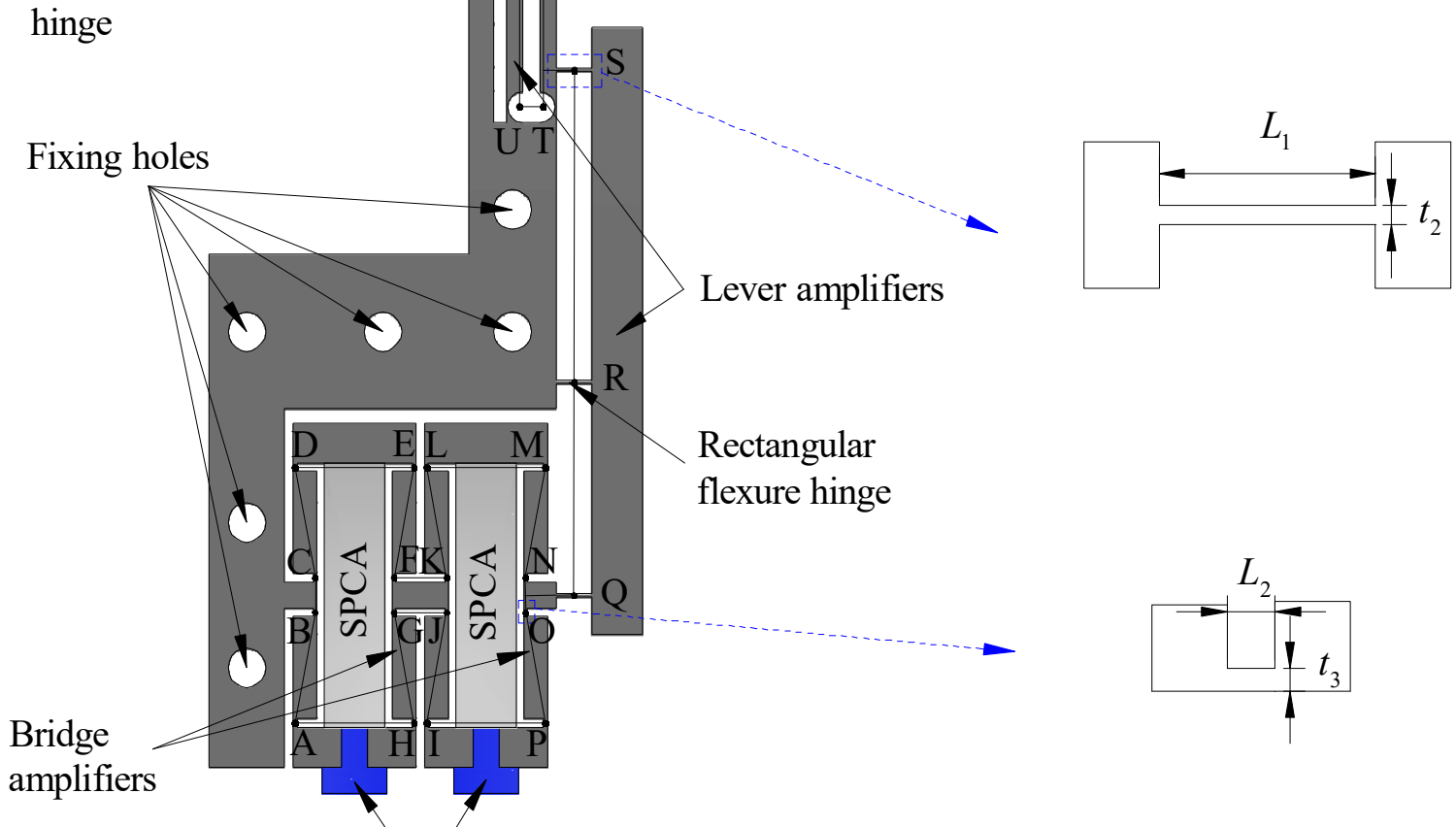

\section{Preload bolts}

Figure 1. Main view of the microgripper. SPCA, stacked piezoelectric (PZT) ceramic actuator.

\subsection{Movement Principle of the Microgripper}

Figure 2 shows the structure diagram of the microgripper, and the corresponding motion principle is shown in the figure. The microgripper consisted of two bridge amplifiers and two lever amplifiers. 


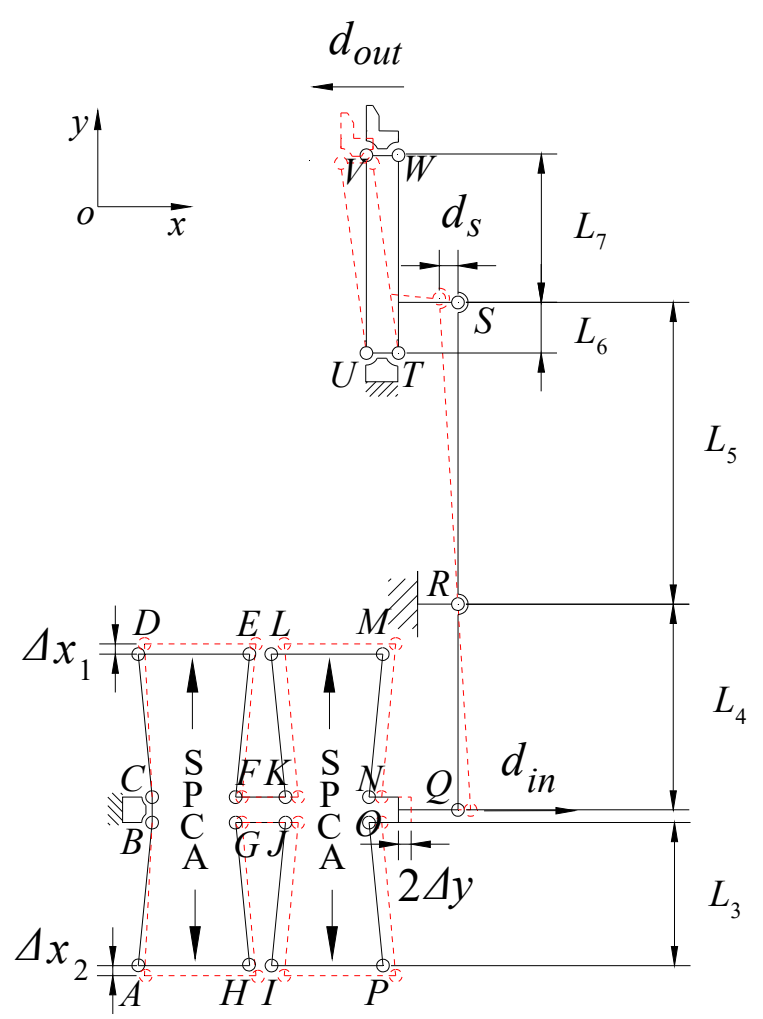

Figure 2. Pseudo rigid body model of the PZT driven microgripper.

Firstly, the bridge amplification mechanism was analyzed. The bridge type amplifier was a symmetrical structure, and the deformation of four side lengths was the same. The stress analysis of one side length was conducted, and the corresponding force analysis diagram is shown in Figure 3. The upper left side length $A B$ of the double bridge amplifier in Figure 2 was analyzed. After the piezoelectric ceramic generated horizontal thrust, point $A$ moved to the left along the horizontal direction to point $A^{\prime}$, and point $B$ moved to the left along the vertical direction at $B^{\prime}$; if the moment of side length $A B$ was counterclockwise, then the moment of side length $A B$ was $2 M_{\mathrm{r}}$.

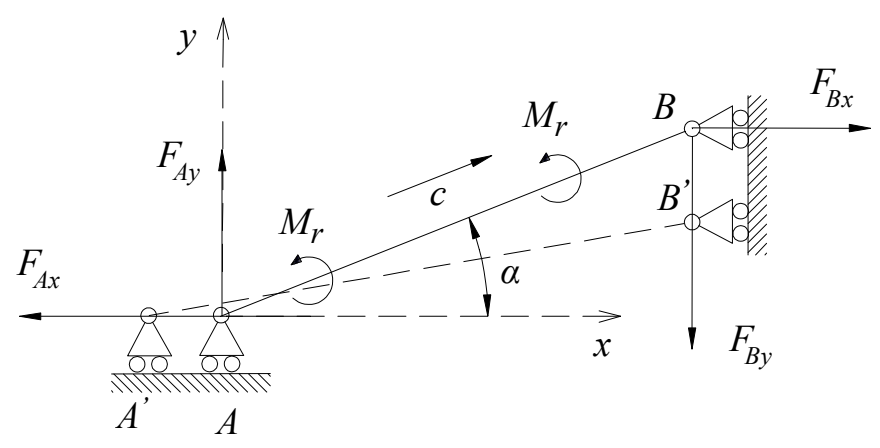

Figure 3. Single side deformation of the bridge amplifier.

According to the stress relationship, the formula can be achieved:

$$
\left\{\begin{array}{l}
F_{A x}=F_{B x}=F_{x}=F_{P S T} / 4 \\
F_{A y}=F_{B y}=F_{y}=F_{P S T} / 4 \\
2 M_{r}=F_{x} L_{A B} \sin \alpha+F_{y} L_{A B} \cos \alpha
\end{array}\right.
$$


A point on side length $A B$ was selected. The distance from this point to point $A$ was $c$, and the corresponding force and moment equations could be obtained:

$$
\left\{\begin{array}{l}
F_{N}=F_{x} \cos \alpha-F_{y} \sin \alpha \\
M=M_{r}-F_{x} c \sin \alpha-F_{y} c \cos \alpha
\end{array}\right.
$$

The deformation energy of side length $A B$ could be divided into two parts: tensile deformation energy $E_{1}$ and bending deformation energy $E_{2}$; thus:

$$
E=E_{1}+E_{2}
$$

According to the second theorem of Castigliano, the deformation of the side length in the $x$ direction can be written as:

$$
\begin{gathered}
\Delta x=\Delta x_{1}+\Delta x_{2} \\
\Delta x=\frac{\partial E}{\partial F_{x}}=\frac{F_{N} L_{A B}}{E A} \frac{\partial F_{N}}{\partial F_{x}}+\int_{0}^{L_{A B}} \frac{M}{E I} \frac{\partial M}{\partial F_{x}} d c=\frac{L_{A B}}{E A}\left(F_{x} \cos \alpha-F_{y} \sin \alpha\right) \cos \alpha+ \\
\frac{L_{A B}{ }^{3}}{12 E I}\left(F_{x} \sin \alpha+F_{y} \cos \alpha\right) \sin \alpha
\end{gathered}
$$

where $E A$ is tensile stiffness and $E I$ is bending stiffness.

$$
\begin{gathered}
\Delta y=\frac{\partial E}{\partial F_{y}}=\frac{F_{N} L_{A B}}{E A} \frac{\partial F_{N}}{\partial F_{y}}+\int_{0}^{L_{A B}} \frac{M}{E I} \frac{\partial M}{\partial F_{y}} d c=\frac{L_{A B}}{E I}\left(F_{x} \cos \alpha-F_{y} \sin \alpha\right) \sin \alpha+ \\
\frac{L_{A B}^{3}}{12 E I}\left(F_{x} \sin \alpha+F_{y} \cos \alpha\right) \cos \alpha
\end{gathered}
$$

The displacement amplification ratio of single bridge amplifier can be written as:

$$
R_{\mathrm{amp} 0}=\frac{\Delta y}{\Delta x}=\frac{\left(L_{A B}{ }^{2}-h^{2}\right) \sin \alpha \cos \alpha}{h^{2} \cos ^{2} \alpha+6 L_{A B^{2}} \sin ^{2} \alpha}
$$

Thus, the displacement amplification ratio of double bridge amplifier is:

$$
R_{\mathrm{amp} 1}=\frac{2 \Delta y}{\Delta x}=\frac{2\left(L_{A B}{ }^{2}-h^{2}\right) \sin \alpha \cos \alpha}{h^{2} \cos ^{2} \alpha+6 L_{A B^{2}} \sin ^{2} \alpha}
$$

The output stage of the compliant parallelogram parallel mechanism on the moveable jaw had a parasitic rotation about the Z-axis, which reduced the gripping accuracy of the jaw [35-37]. However, the parasitic rotation was much smaller than the principal motion, the translation along the $\mathrm{X}$-axis. It could be calculated from finite element analysis that the rotation displacement was about $0.0658 \%$ of the gripping displacement, as shown in Figure 4 . Therefore, the parasitic rotation could be ignored in this case, while it could also be eliminated by applying the actuation force along the stiffness center of the compliant parallelogram mechanism (shown in Figure $4 \mathrm{~b}$ ) in other cases if a larger geometric dimension was not a problem.

Based on the pseudo rigid body model (PRBM) method, the analysis of the double lever amplifier was carried out. The PRBM of the mechanism is shown in Figure 5. Displacement $d_{\text {in }}$ was applied to the input end, and the first displacement amplification was realized by the lower lever amplifier. The output displacement $s$ of the lower lever amplifier can be expressed as:

$$
d_{s}=\frac{\left(l_{2}+l_{3}\right) d_{i n}}{l_{1}}
$$




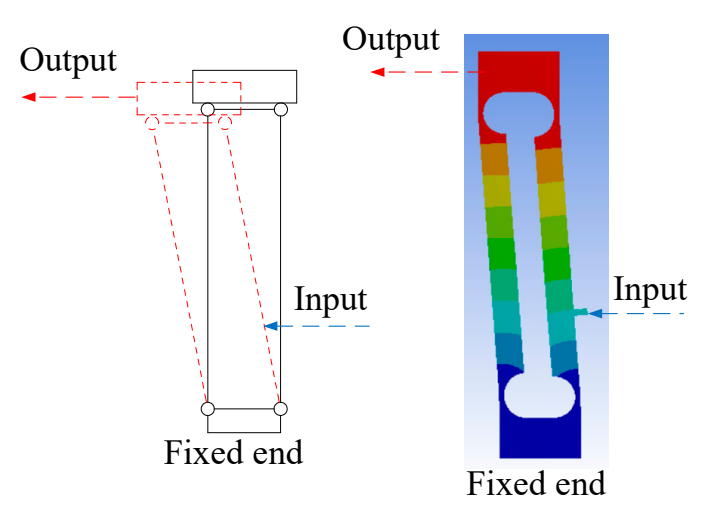

(a)

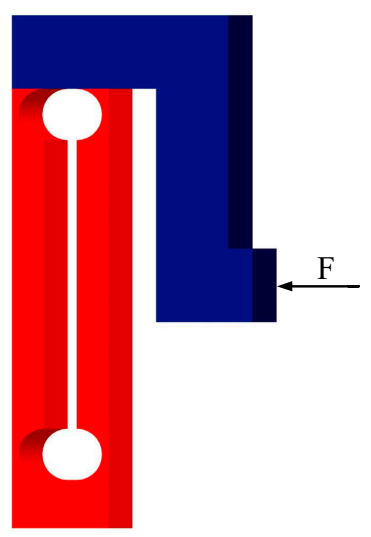

(b)

Figure 4. Amplification principle of the parallelogram mechanism: (a) traditional parallelogram mechanism and $(\mathbf{b})$ a new type of parallelogram mechanism.

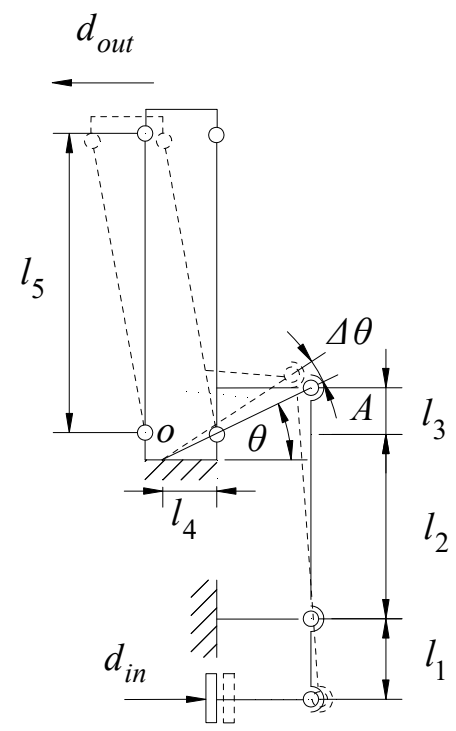

Figure 5. Pseudo rigid body model (PRBM) of double lever amplifiers.

The movement of the lower lever amplifier drove the upper lever amplifier to undergo secondary deformation. When the mechanism was not deformed, the angle between the line $O A$ and the horizontal direction was $\theta$. After the mechanism was deformed, the change in the amount of the angle $\theta$ was $\Delta \theta$. Then, there was the following geometric relationship:

$$
\begin{gathered}
d_{\text {in }}=\frac{l_{1} \sqrt{l_{3}^{2}+l_{4}^{2}}}{l_{2}+l_{3}}[\cos (\theta)-\cos (\theta+\Delta \theta)] \\
d_{\text {out }}=l_{5}[\sin (\theta+\Delta \theta)-\sin (\theta)]
\end{gathered}
$$

In the process of movement, the flexure hinge produced small deformation, and the maximum deformation was usually no more than one thousandth of its own size; then $\Delta \theta \approx 0$, so the displacement amplification of the mechanism can be expressed as:

$$
R_{\mathrm{amp} 2}=\frac{d_{\text {out }}}{d_{\text {in }}}=\frac{l_{5}\left(l_{2}+l_{3}\right)}{l_{1} \sqrt{l_{3}^{2}+l_{4}^{2}}}
$$


Therefore, the amplification of the four stage amplification microgripper is:

$$
R_{\mathrm{amp}}=R_{\mathrm{amp} 1} R_{\mathrm{amp} 2}=\frac{2 \Delta y d_{\text {out }}}{\Delta x d_{\text {in }}}=\frac{2 l_{5}\left(l_{2}+l_{3}\right)\left(L_{A B}{ }^{2}-h^{2}\right) \sin \alpha \cos \alpha}{l_{1} \sqrt{l_{3}^{2}+l_{4}^{2}}\left(h^{2} \cos ^{2} \alpha+6 L_{A B}^{2} \sin ^{2} \alpha\right)}
$$

\section{Finite Element Analysis}

\subsection{Size Parameter Optimization}

The structure diagram of the microgripper is shown in Figure 1. The performance of the microgripper was mainly determined by the structure size of the hinges. Based on the ANSYS software response surface method, the structure size of the microgripper hinges was optimized. In order to obtain larger displacement amplification, the output displacement was chosen as the optimization objective function. The key parameters $a, b, c, d, e, f, g, h$ were selected as input variables, which were defined according to practical application and manufacturing conditions within a certain range. The maximum stress in the working process should be less than the yield strength of the material. In order to improve the performance of the microgripper, the optimization design was carried out as follows:

(1) Objective: Maximum output displacement.

(2) Related parameters: $n, t_{3}, b_{1}, b_{w}, m, t_{2}, d, e, f, g, r, t_{1}$.

(3) Subject to:

(a) constraint equations: $\sigma_{\max }=\frac{(\sigma)}{n_{a}}$, where $(\sigma)$ denotes the tensile yield stress and $n_{a}=2$ represents the factor of safety;

(b) parameter range: $0.4 \mathrm{~mm} \leq n \leq 0.8 \mathrm{~mm}, 0.2 \mathrm{~mm} \leq t_{3} \leq 0.4 \mathrm{~mm}, 6 \mathrm{~mm} \leq b_{l} \leq 8 \mathrm{~mm}, 1.5 \mathrm{~mm} \leq$ $b_{w} \leq 2.5 \mathrm{~mm}, 2.5 \mathrm{~mm} \leq m \leq 3.5 \mathrm{~mm}, 0.2 \mathrm{~mm} \leq t_{2} \leq 0.4 \mathrm{~mm}, 14 \mathrm{~mm} \leq d \leq 18 \mathrm{~mm}, 20 \mathrm{~mm} \leq e \leq 25 \mathrm{~mm}$, $3 \mathrm{~mm} \leq f \leq 5 \mathrm{~mm}, 10 \mathrm{~mm} \leq g \leq 12 \mathrm{~mm}, 0.8 \mathrm{~mm} \leq r \leq 1.8 \mathrm{~mm}, 0.2 \mathrm{~mm} \leq t_{1} \leq 0.4 \mathrm{~mm}$.

The optimal geometric parameters of the microgripper could be obtained by looking at the trade-off diagram and achieve the optimal performance. The optimized size is shown in Table 1.

Table 1. Optimized dimensions.

\begin{tabular}{ccc}
\hline Parameter & Description & Value \\
\hline$L_{2} \times t_{3}$ & Dimensions of rectangular flexure hinges & $0.64 \times 0.28 \mathrm{~mm}^{2}$ \\
$L_{3}$ & Dimensions of the side length of the bridge amplifier & $7.72 \times 1.88 \mathrm{~mm}^{2}$ \\
$L_{1} \times t_{2}$ & Dimensions of rectangular flexure hinges & $2.87 \times 0.28 \mathrm{~mm}^{2}$ \\
$L_{4}$ & Dimension of the lever short arm & $16.12 \mathrm{~mm}$ \\
$L_{5}$ & Dimension of the lever long arm & $23.64 \mathrm{~mm}$ \\
$L_{6}$ & Dimension of the lever short arm & $3.96 \mathrm{~mm}$ \\
$L_{7}$ & Dimension of the lever long arm & $11.37 \mathrm{~mm}$ \\
$R \times t_{1}$ & Dimension of asymmetric right-circular flexure hinges & $1.18 \times 0.28 \mathrm{~mm}^{2}$ \\
\hline
\end{tabular}

\subsection{Performance Analysis of Microgripper}

The finite element analysis software ANSYS (15.0) was used to simulate the deformation of the microgripper. The material of the microgripper and micro-parts in the analysis process was 7075 aluminum alloy, a modulus of elasticity $E=71 \mathrm{GPa}$, a Poisson's ratio $v=0.33$, a yield strength $\sigma=455 \mathrm{MPa}$, and a density $\rho=2810 \mathrm{~kg} / \mathrm{m}^{3}$. In the process of simulation, the 3D solid model built in SolidWorks was imported into ANSYS for mesh generation; constraints and static loads were applied; and static analysis was carried out. For the flexible part of the flexure hinges of the microgripper, we set a small mesh generation parameter $(0.1 \mathrm{~mm})$, and the other parts generated the mesh automatically, which could not only accurately describe these parameters to improve the accuracy of the analysis results, but also accelerate the analysis speed and save time; the constraint conditions and static load application position are shown in Figure 6a. 


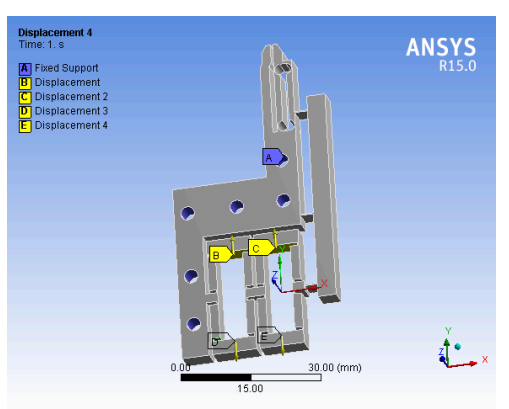

(a)

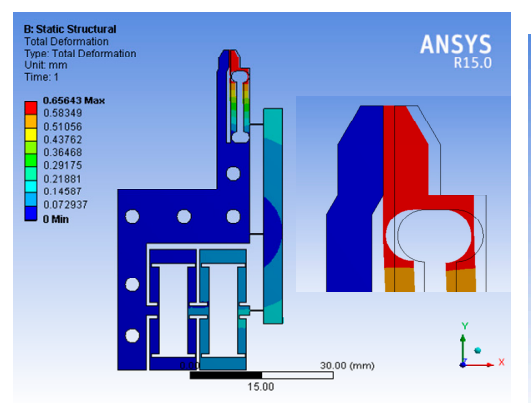

(b)

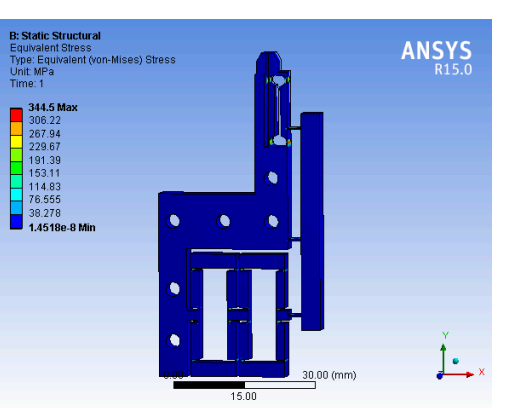

(c)

Figure 6. FEA diagram of the microgripper: (a) FEA settings, (b) deformation pattern and (c) stress pattern.

Figure $6 \mathrm{~b}, \mathrm{c}$ is the corresponding displacement nephogram of the microgripper when $20 \mu \mathrm{m}$ of input displacement was applied to the input end and the microparts did not grip. Figure $6 \mathrm{~b}$ is the displacement nephogram of the microgripper. It can be clearly seen that during the closing process of the microgripper, the right jaw gripped in parallel; the maximum output displacement of one side was $656.43 \mu \mathrm{m}$; the displacement amplification was 32.82 times. Figure $6 \mathrm{c}$ is the strain nephogram of the microgripper, from which it can be seen that the maximum stress was $344.5 \mathrm{MPa}$, which was less than the yield strength of the material, so the product could be used safely.

The grasping force was estimated by FEA. Figure 7 shows the SPCA input force versus the output force of the gripper when the micro-axes of $600 \mu \mathrm{m}, 450 \mu \mathrm{m}$, and $300 \mu \mathrm{m}$ were gripped by the microgripper, respectively, where $D$ is the initial distance between the jaws, $d$ is the micro-object diameter, and $s$ is the distance between the jaw and micro-objects. It can be seen from the figure that the linearity of the input displacement and the output force was large and easy to control, and a smooth transition was achieved from the closing of the grasping jaw to the gripping of the micro-axis. When the micro-axis of $600 \mu \mathrm{m}$ was gripped, the theoretical output force of the gripper jaw was 270 $\mathrm{mN}$ under the input displacement of $22 \mathrm{~N}$.

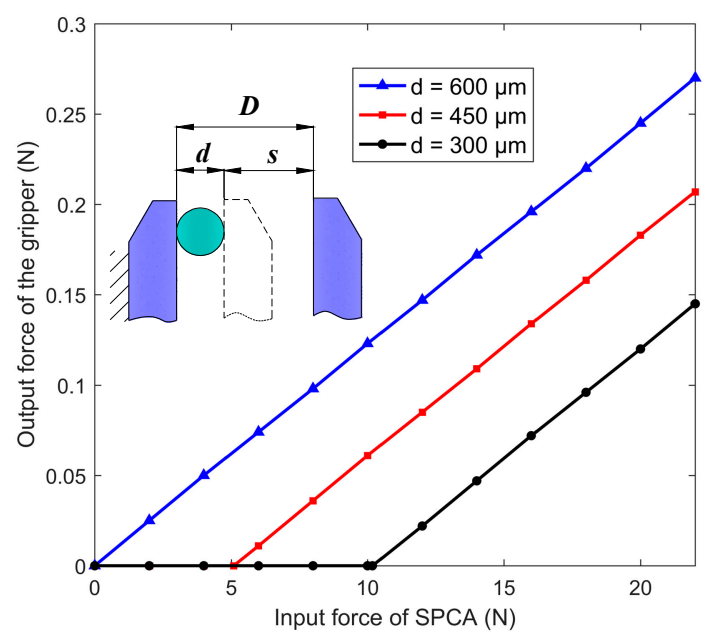

Figure 7. SPCA input force versus the output force of the gripper.

\section{Experiments}

\subsection{Physical Model of Microgripper}

The physical model of the microgripper is shown in Figure 8. The material used for the microgripper was 7075-T6 (SN) aluminum alloy. The microgripper was processed by the wire 
electrical-discharge machining (WEDM) with slow walking. After processing, the microgripper was drilled and polished. The experimental equipment included the HPV-1 C 0300 A0300 piezoelectric ceramic driving power supply (Suzhou Mat Inc., Suzhou, China), micro-sodium positioning worktable (Suzhou Mat Inc., Suzhou, China), PZT (SZBS150/5 × 5/20, open-loop travel 20 m, Suzhou Mat Inc., Suzhou, China) driving micro-positioning stage (Suzhou Mat Inc., Suzhou, China), high resolution capacitive displacement sensor (BJZD Company MA-0.5, Beijing, China), data acquisition card (NI Company PCI-6221, Dongwan, China), 24 V DC regulated power supply WP100-D-G (Suzhou Mat Inc., Suzhou, China), host computer and display matched with the sensor signal amplifier BSQ-2 (Suzhou Mat Inc., Suzhou, China), and 24 V DC regulated power supply WP100-D-G. In order to eliminate the external interference as much as possible, all the devices were installed on the high performance vibration isolation platform (ZPT-G-M, zh-gezhen, Wuhan, China).

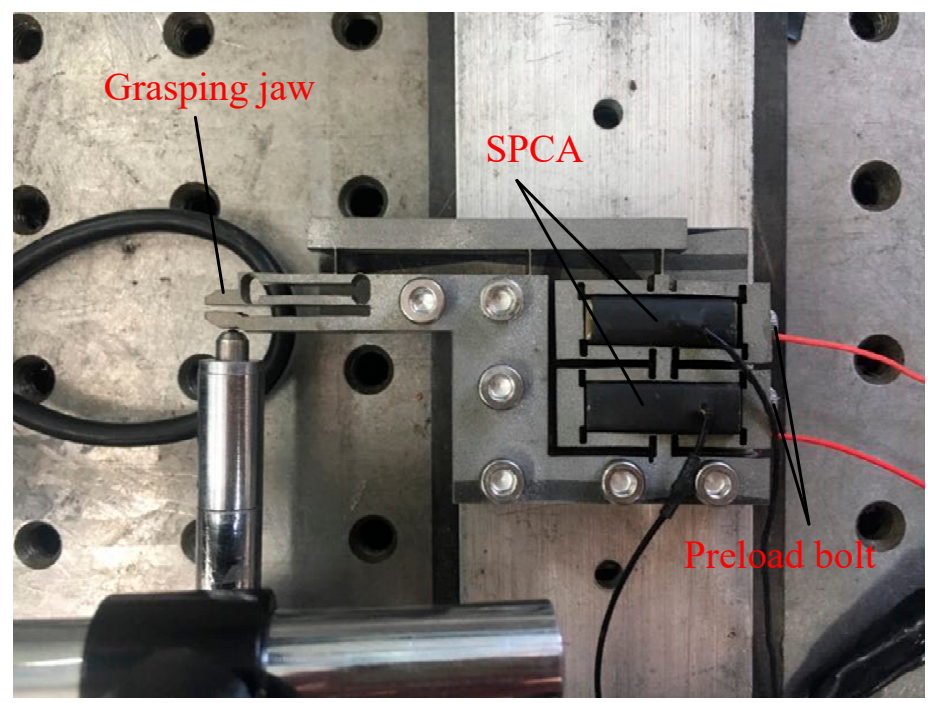

Figure 8. Physical model of the microgripper.

\subsection{Experimental Verification}

In order to further test the performance of the microgripper, a series of experiments was carried out. The experimental device is shown in Figure 9. Figure 10a is the functional block diagram of the experimental device, and Figure $10 \mathrm{~b}$ is the actual working diagram of the experimental device.
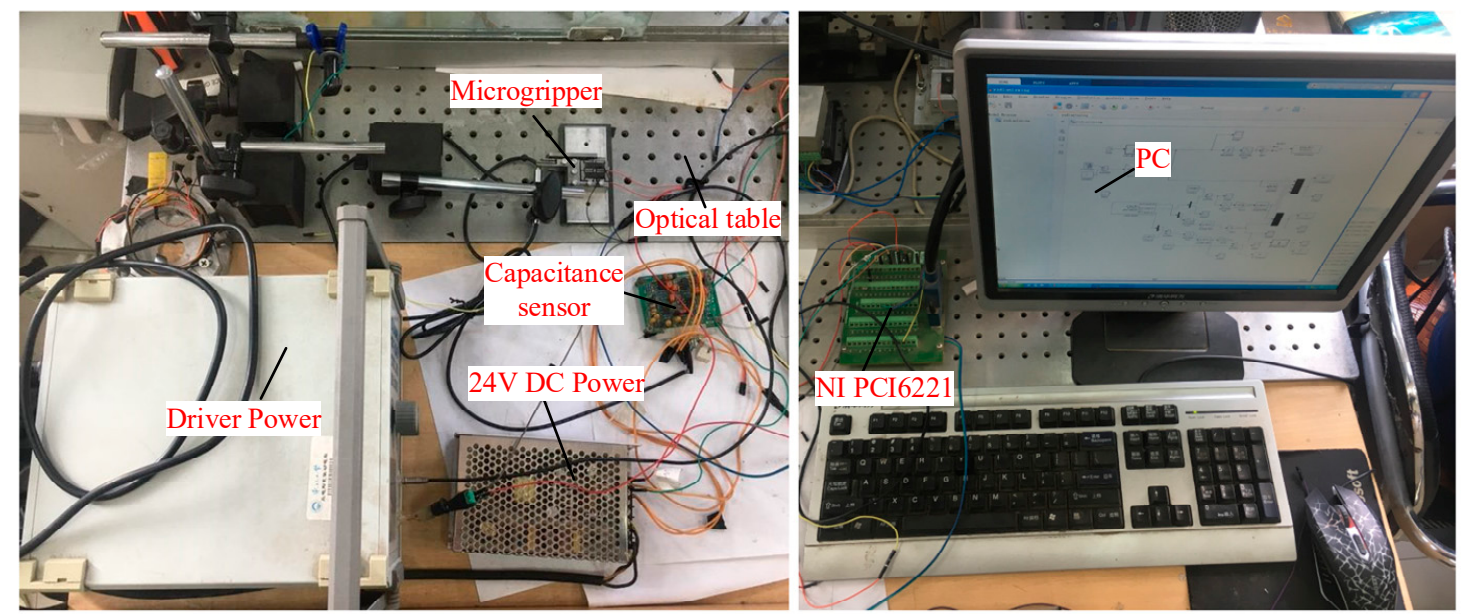

Figure 9. Experimental setup of the microgripper. 


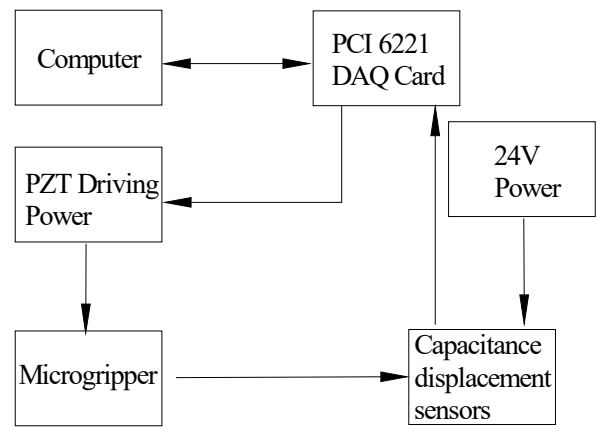

(a)

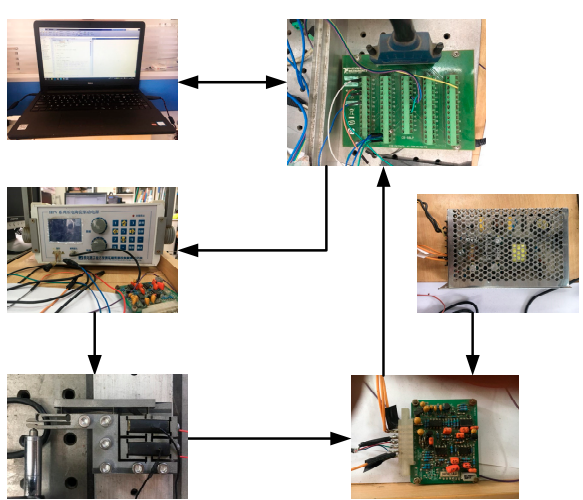

(b)

Figure 10. Working diagram of the experimental device. (a) functional block diagram and (b) actual working diagram.

Figure 11 shows the relationship between the input displacement and the piezoelectric actuator driving voltage from 0 to $150 \mathrm{~V}$. The results confirmed the nonlinear characteristic and hysteresis of the piezoelectric material. When the input voltage was $150 \mathrm{~V}$, the maximum output displacement was $22.08 \mu \mathrm{m}$, which was larger than the nominal output displacement of SPCA. Under the open-loop control condition, when the driving voltage was $20 \mathrm{~V}$, the output displacement difference of expansion and contraction reached the maximum value, which was $2.74 \mu \mathrm{m}$.

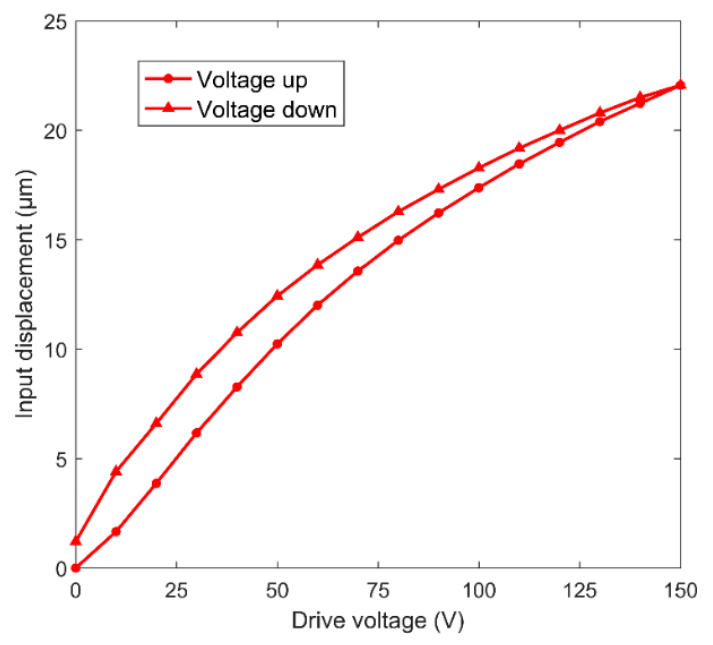

Figure 11. Input displacement versus the drive voltage.

However, the nonlinearity of the piezoelectric material did not affect the linear relationship between the input and output of the microgripper. Figure 12 shows the SPCA input displacement versus the tip displacement of the grasping jaw. From the graph, it can be seen that the input displacement of SPCA was linearly related to the tip displacement of the grasping jaw, which showed that the microgripper had a stable performance.

The theoretical amplification factor was 34.65 times; the FEA simulation amplification factor was 32.82 times; and the experimental amplification factor was 31.6 times. The error between the experiment and the FEA simulation was $5.28 \%$, while the error between the experiment and the theoretical calculation was $8.80 \%$. The three displacement amplification ratios by different methods were very close, which proved the correctness of the theoretical calculation. There was a certain degree of deviation between the physical model and the theoretical modeling due to the machining error, which resulted in the error between the experiment value, FEA simulation value, and theoretical calculation value. The idea of theoretical calculation is based on geometric relation, which only 
considers the deformation of the flexure hinges and regards the other parts of the flexure hinge as rigid bodies. Actually, there was indeed deformation in other parts of the flexure hinges. On the other hand, the multi-stage amplifier could restrain the single machine amplifier; that is why the theoretical calculation value was larger than the FEA simulation value and the experiment value. The accuracy error in the process of microgripper machining, the measurement error in the process of the experiment, and the influence of equipment vibration and noise were inevitable; that was why the experiment value was smaller than the FEA simulation value.

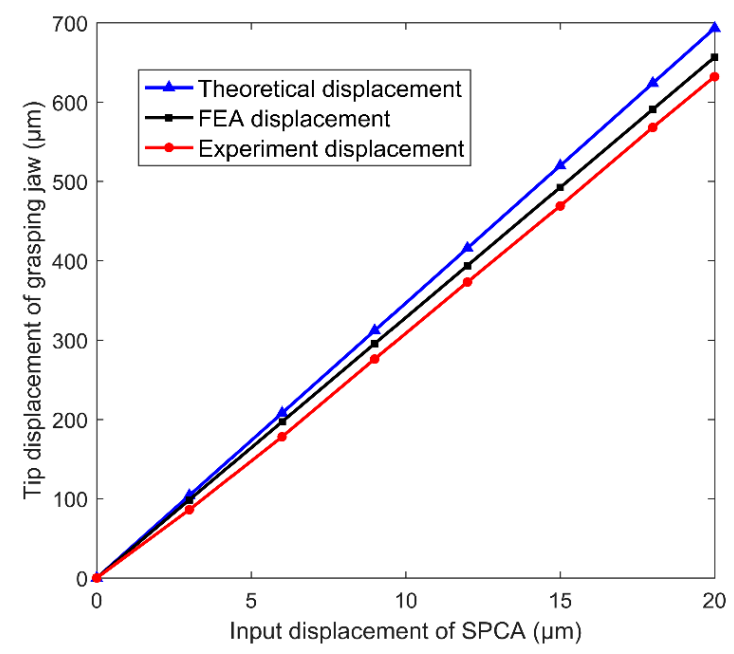

Figure 12. SPCA input displacement versus the tip displacement of the grasping jaw.

The experimental results of displacement amplification are shown in Table 2. The input displacement and output displacement were measured by a displacement sensor. In order to further verify the performance of the microgripper, the test device was started and closed five times. Under the action of $150 \mathrm{~V}$ driving voltage, the input displacement of SPCA and the tip displacement of grasping jaw were measured five times. The average value of the five displacement magnifications $k=31.6$, and the standard deviation was 0.019 . The experimental results proved the stability of the microgripper.

Table 2. Experiment results of displacement amplification.

\begin{tabular}{ccccc}
\hline No. & Driven Power (V) & $\begin{array}{c}\text { Input Displacement } \\
(\boldsymbol{\mu m})\end{array}$ & $\begin{array}{c}\text { Tip Displacement } \\
(\boldsymbol{\mu m})\end{array}$ & $k$ \\
\hline 1 & 150 & 22.06 & 697.1 & 31.60 \\
2 & 150 & 21.93 & 693.65 & 31.63 \\
3 & 150 & 22.15 & 699.94 & 31.60 \\
4 & 150 & 22.18 & 700.44 & 31.58 \\
5 & 150 & 21.97 & 694.03 & 31.59 \\
\hline
\end{tabular}

In order to verify the applicability of the microgripper in the operation of micro-objects with different shapes and sizes, several grasping experiments were carried out. Figure 13 shows the microgripper grasping metal plate, wire, and plastic plate, respectively. The results showed that the microgripper had the characteristics of parallel gripping and a large grasping range for different shapes of objects and could successfully grasp micro-objects. Figure 14 shows the microgripper parallel grasping a $250 \mu \mathrm{m}$ metal ball. 


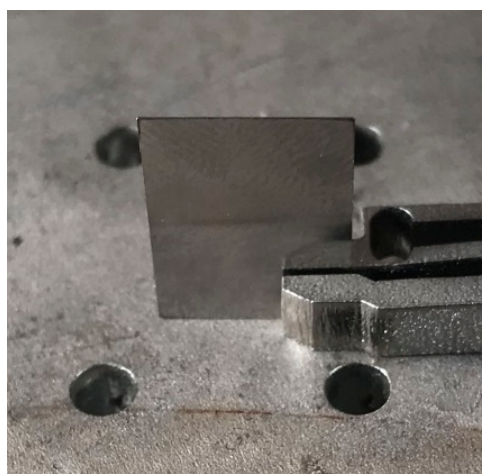

(a)

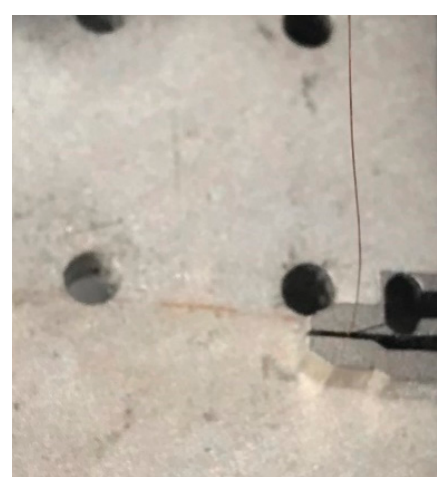

(b)

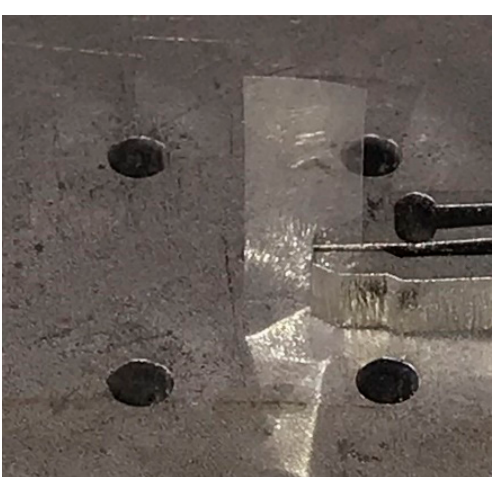

(c)

Figure 13. Grasping manipulation for different shaped and sizes of micro-objects (from left to right): (a) $300 \mu \mathrm{m}$ metal plate, (b) $250 \mu \mathrm{m}$ metal wire and (c) $120 \mu \mathrm{m}$ plastic plate.

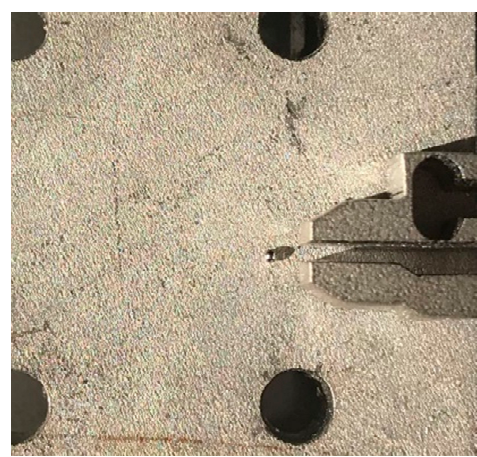

(a)

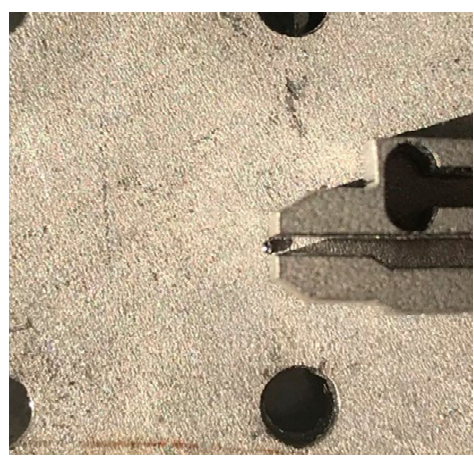

(b)

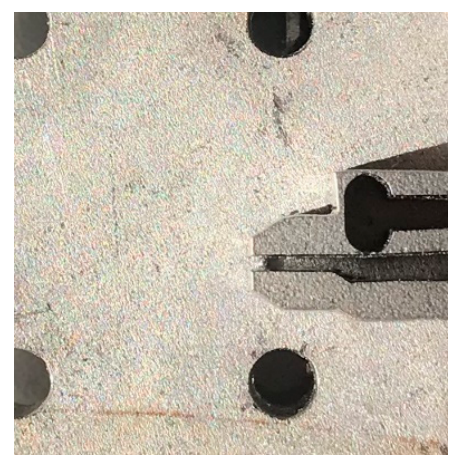

(c)

Figure 14. (a,b) Approaching and (c) grasping the micro-object.

\subsection{Performance Comparison}

The amplification of our microgripper versus similar devices [13-19] is shown in Table 3. The amplification of the microgripper designed in this paper reached 31.6 times, which was greater than other microgrippers.

Table 3. Comparison of the parameters of similar microgrippers.

\begin{tabular}{ccc}
\hline Microgripper & Single Side Amplification & Total Amplification \\
\hline Reference [24] & - & 22.89 \\
Reference [25] & - & 6.88 \\
Reference [26] & - & 16.4 \\
Reference [27] & - & 22.6 \\
Reference [28] & 3.68 & 3.68 \\
Reference [29] & 4.16 & 4.16 \\
Reference [30] & 6.1 & 6.1 \\
Reference [31] & 14.94 & 14.94 \\
Design of this paper & 31.6 & 31.6 \\
\hline
\end{tabular}

\section{Conclusions}

To address the disadvantages of the existing symmetrical microgrippers, this paper proposed a new PZT actuated microgripper. A large displacement amplification ratio was achieved for the proposed microgripper by using a four stage flexure based amplification. The new microgripper not only had large output displacement, the same as the traditional symmetrical microgrippers, but also possessed stable gripping performance. The relationship between the input and output displacements 
of the microgripper was obtained through theoretical derivation and verified by FEA simulations. A prototype of the microgripper was manufactured by WEDM with slow walking, and experimental tests were carried out on the prototype. The experimental results matched well with both the theoretical results and the simulation results. The microgripper designed in this paper could achieve high magnification displacement up to 31.6 and could operate on micro-parts of any irregular shapes smaller than $632 \mu \mathrm{m}$ in size. In this paper, a series of gripping experiments was performed on the microgripper, and a variety of irregularly shaped micro-parts were successfully gripped without any damage. The design principle of the compliant mechanism based microgripper provided a useful reference for research on multi-stage amplification manipulators and microgrippers.

Author Contributions: Conceptualization, X.C.; methodology, X.C. and Z.D.; software, X.C.; validation, X.C and J.G.; formal analysis, S.H.; investigation, Z.D.; resources, Z.D.; data curation, S.H.; writing-original draft preparation, X.C.; writing-review and editing, X.C.; project administration, X.G.; funding acquisition, X.G. All authors have read and agreed to the published version of the manuscript.

Funding: This research was funded by the Liaoning Provincial Department of Education Project (L2017LQN024).

Conflicts of Interest: The authors declare no conflict of interest.

\section{References}

1. Lyshevski, S.E. Nano-and Micro-Electromechanical Systems: Fundamentals of Nano- and Microengineering; CRC Press: Boca Raton, FL, USA, 2018.

2. Zhang, S.J.; To, S.; Wang, S.J.; Zhu, Z.W. A review of surface roughness generation in ultra-precision machining. Int. J. Mach. Tools Manuf. 2015, 91, 76-95. [CrossRef]

3. Madl, C.M.; Heilshorn, S.C.; Blau, H.M. Bioengineering strategies to accelerate stem cell therapeutics. Nature 2018, 557, 335-342. [CrossRef]

4. Kim, I.; Kim, M.-H.; Lim, S. Reproductive hazards still persist in the microelectronics industry: Increased risk of spontaneous abortion and menstrual aberration among female workers in the microelectronics industry in South Korea. PLoS ONE 2015, 10, e0123679. [CrossRef]

5. Jiang, J.; Wang, S.; Liu, K.; Zhang, X.; Jinde, Y.; Wu, F.; Liu, T. Development of optical fiber temperature sensor for aviation industry. In Proceedings of the 2016 15th International Conference on Optical Communications and Networks (ICOCN), Hangzhou, China, 24-27 September 2016.

6. Yong, Y.K.; Bhikkaji, B.; Moheimani, S.O.R. Design, modeling, and FPAA-based control of a high-speed atomic force microscope nanopositioner. IEEE/ASME Trans. Mechatron. 2013, 18, 1060-1071. [CrossRef]

7. Guo, Z.; Tian, Y.; Liu, X.; Wang, F.; Zhou, C.; Zhang, D. Experimental investigation of the tip based micro/nano machining. Appl. Surf. Sci. 2017, 426, 406-417. [CrossRef]

8. Nachippan, N.M.; Venkatesh, A.P.; Muniyappan, M. Modelling and analysis of piezoelectric microgripper for unmanned aerial vehicle. Mater. Today Proc. 2018, 5, 19456-19462. [CrossRef]

9. Shi, H.; Shi, W.; Zhang, R.; Zhai, J.; Chu, J.; Dong, S. A micromachined piezoelectric microgripper for manipulation of micro/nanomaterials. Rev. Sci. Instrum. 2017, 88, 065002. [CrossRef] [PubMed]

10. Boudaoud, M.; Gorrec, Y.L.; Haddab, Y.; Lutz, P. Gain scheduling control of a nonlinear electrostatic microgripper: Design by an eigenstructure assignment with an observer-based structure. IEEE Trans. Control Syst. Technol. 2015, 23, 1255-1267. [CrossRef]

11. Phelan, M.; Furlong, C. Characterization of a MEMS Electrostatic Microgripper for Micromanipulation and Sensing. In Micro and Nanomechanics; Springer: Cham, Germany, 2018; Volume 5, pp. 65-72.

12. Zandi, A.M.; Wang, C.; Voicu, R.-C.; Muller, R. Testing and characterisation of electrothermal microgrippers with embedded microheaters. In Proceedings of the 2016 Symposium on Design, Test, Integration and Packaging of MEMS/MOEMS (DTIP), Budapest, Hungary, 30 May-2 June 2016.

13. Al-Zandi, M.H.M.; Wang, C.; Voicu, R.; Muller, R. Measurement and characterisation of displacement and temperature of polymer based electrothermal microgrippers. Microsyst. Technol. 2018, 24, 379-387. [CrossRef]

14. Chang, R.-J.; Lai, Y.-H. Design and implementation of micromechatronic systems: SMA drive polymer microgripper. In Design, Control and Applications of Mechatronic Systems in Engineering; IntechOpen: London, UK, 2017; p. 65. 
15. Munasinghe, K.C.; Bowatta, B.G.C.T.; Abayarathne, H.Y.R.; Kumararathna, N.; Maduwantha, L.K.A.H.; Arachchige, N.M.P.; Amarasinghe, Y.W.R. New MEMS based micro gripper using SMA for micro level object manipulation and assembling. In Proceedings of the 2016 Moratuwa Engineering Research Conference (MERCon), Moratuwa, Sri Lanka, 5-6 April 2016.

16. Choi, A.; Gultepe, E.; Gracias, D.H. Pneumatic delivery of untethered microgrippers for minimally invasive biopsy. In Proceedings of the 2017 13th IEEE International Conference on Control \& Automation (ICCA), Ohrid, Macedonia, 3-6 July 2017.

17. Gursky, B.; Bütefisch, S.; Leester-Schädel, M.; Li, K.; Matheis, B. A disposable pneumatic microgripper for cell manipulation with image-based force sensing. Micromachines 2019, 10, 707. [CrossRef]

18. $\mathrm{Xu}, \mathrm{Q}$. Adaptive discrete-time sliding mode impedance control of a piezoelectric microgripper. IEEE Trans. Robot. 2013, 29, 663-673. [CrossRef]

19. Nkoonbin, A.; Hassani Niaki, M. Deriving and analyzing the effective parameters in microgrippers performance. Sci. Iran. 2012, 19, 1554-1563.

20. Chen, W. Research on Piezoelectric Driven Microgripper Based on Compliant Mechanisms. Ph.D. Thesis, South China University of Technology, Guangzhou, China, 2017.

21. Chen, W.; Shi, X.; Chen, W.; Zhang, J. A two degree of freedom micro-gripper with grasping and rotating functions for optical fibers assembling. Rev. Sci. Instrum. 2013, 84, 115111. [CrossRef]

22. Blideran, M.M.; Bertsche, G.; Henschel, W.; Kern, D.P. A mechanically actuated silicon microgripper for handling micro- and nanoparticles. Microelectron. Eng. 2006, 83, 1382-1385. [CrossRef]

23. Sun, L.; Chen, L.; Rong, W. Key technologies of micromanipulation equipment for microelectromechanical system assembly and packaging. J. Mech. Eng. 2008, 44, 13-19. [CrossRef]

24. Li, Z.; Sun, B.; Yang, G. Design of a microgripper based on topology optimization. J. Dalian Univ. Technol. 2005, 45, 210-214.

25. Cui, Y.G.; Zhu, Y.X.; Lou, J.Q. Detection of finger displacement and clamping force of piezoelectric micro-gripper. Opt. Precis. Eng. 2015, 23, 1372-1379. [CrossRef]

26. Sun, X.; Chen, W.; Fatikow, S.; Tian, Y.; Zhou, R.; Zhang, J.; Mikczinski, M. A Novel piezo-driven microgripper with a large jaw displacement. Microsyst. Technol. 2015, 21, 931-942. [CrossRef]

27. Wang, F.; Liang, C.; Tian, Y.; Zhao, X.; Zhang, D. Design and control of a compliant microgripper with a large amplification ratio for high-speed micro manipulation. IEEE/ASME Trans. Mechatron. 2016, 21, 1262-1271. [CrossRef]

28. Koo, B.W.; Hong, S.P.; Kim, S.I.; Kang, C.S.; Han, S.S.; Oh, K.H.; Kim, Y.W. Design and application of a novel in situ nano-manipulation stage for transmission electron microscopy. Microsc. Microanal. 2015, 21, $298-306$. [CrossRef]

29. Xing, Q. Design of asymmetric flexible micro-gripper mechanism based on flexure hinges. Adv. Mech. Eng. 2015, 7, 1-8.

30. Zhang, D.; Zhang, Z.; Gao, Q.; Xu, D.; Liu, S. Development of a monolithic compliant SPCA-driven micro-gripper. Mechatronics 2015, 25, 37-43. [CrossRef]

31. Liang, C.; Wang, F.; Shi, B.; Huo, Z.; Zhou, K.; Tian, Y.; Zhnag, D. Design and control of a novel asymmetrical piezoelectric actuated microgripper for micromanipulation. Sens. Actuators A Phys. 2018, 269, 227-237. [CrossRef]

32. Zelenika, S.; Munteanu, M.G.; De Bona, F. Optimized flexural hinge shapes for microsystems and high-precision applications. Mech. Mach. Theory 2009, 44, 1826-1839. [CrossRef]

33. Linß, S.; Schorr, P.; Zentner, L. General design equations for the rotational stiffness, maximal angular deflection and rotational precision of various notch flexure hinges. Mech. Sci. 2017, 8, 29. [CrossRef]

34. Valentini, P.P.; Pennestrì, E. Elasto-kinematic comparison of flexure hinges undergoing large displacement. Mech. Mach. Theory 2017, 110, 50-60. [CrossRef]

35. Awtar, S.; Slocum, A.H. Closed-form nonlinear analysis of beam-based flexure modules. In Proceedings of the ASME 2005 International Design Engineering Technical Conferences and Computers and Information in Engineering Conference, Long Beach, CA, USA, 24-28 September 2005; pp. 101-110. 
36. Luo, Y.; Liu, W.; Wu, L. Analysis of the displacement of lumped compliant parallel-guiding mechanism considering parasitic rotation and deflection on the guiding plate and rigid beams. Mech. Mach. Theory 2015, 91, 50-68. [CrossRef]

37. Gräser, P.; Linß, S.; Zentner, L.; Theska, R. Design and experimental characterization of a flexure hinge-based parallel four-bar mechanism for precision guides. In Microactuators and Micromechanisms; Springer: Cham, Germany, 2017; pp. 139-152.

(c)

(C) 2019 by the authors. Licensee MDPI, Basel, Switzerland. This article is an open access article distributed under the terms and conditions of the Creative Commons Attribution (CC BY) license (http://creativecommons.org/licenses/by/4.0/). 